> La production de connaissances et sa reconnaissance par la communauté scientifique sont des critères incontournables de l'évaluation de la qualité d'une recherche. Les indicateurs bibliométriques de plus en plus utilisés par les décideurs pour orienter leur stratégie font toujours l'objet de nombreux débats. Dans cet article, nous analysons la performance de la France en utilisant principalement deux indicateurs sélectifs obtenus à partir de la base de l'Institute for Scientific Information (ISI): le nombre absolu d'articles et la proportion publiée, dans les journaux ayant un très fort impact - supérieur à 20 ainsi que dans le Top 1 \% des articles biomédicaux les plus cités. À partir de ces données, nous identifions les domaines dominants, les sites les plus actifs pour chacune des spécialités et le réseau de collaborations nationales et internationales. Nos résultats montrent que la recherche biomédicale française contribue fortement aux avancées scientifiques mondiales et que sa qualité atteint les standards internationaux les plus élevés. <

La performance de la France dans le domaine de la recherche fait actuellement l'objet de nombreuses discussions. Les données bibliométriques issues des bases de publications et des observatoires scientifiques peuvent nous donner des éléments objectifs de comparaison. Une première approche se fonde sur la production scientifique de chaque pays soit en nombre de publications, soit en termes de «visibilité ou impact » mesurés par l'ensemble des citations. Les publications scientifiques françaises en 2001, tous domaines confondus, représentent un peu plus de $5 \%$ du

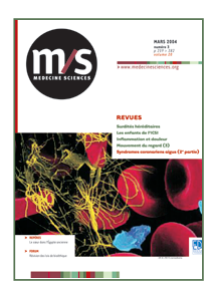

Cellule de Bibliométrie, Département de l'Évaluation scientifique, Inserm, 101 , rue de Tolbiac,

nombre de publications du 75654 Paris Cedex 13, France. monde et correspondent à haeffner@tolbiac.inserm.fr $6,4 \%$ des adresses répertoriées $[1,2]$. Les résultats des différentes études bibliométriques convergent et montrent que la France occupe, en nombre absolu de publications, la $5^{\mathrm{e}}$ place dans le monde et la $3^{e}$ place en Europe, après l'Angleterre et l'Allemagne. Ce classement est identique pour les recherches biomédicales ${ }^{1}$ et non biomédicales $^{2}$ (Tableau 1). Si l'on compare deux périodes, 1997-2001 et 1998-2002, le nombre de publications dans le monde progresse de 3,1\%. Dans le même temps, la part des publications de l'Europe des 15 augmente de 2,8\% et celles de l'Allemagne de $2,7 \%$, de la France de 1,6\%, des États-Unis de 1,5\% et du Royaume-Uni de 1,4 \% [2]. Une analyse, toutes disciplines confondues, de la visibilité de la recherche par la communauté scientifique, mesurée par le nombre total de citations des dix dernières années, place également la France en $5^{\mathrm{e}}$ position mondiale et à la $3^{e}$ place européenne. À noter que dans le domaine biomédical, elle occupe la $4^{\mathrm{e}}$ position mondiale et la $2^{\mathrm{e}}$ place européenne.

${ }^{1}$ Biologie, génétique, neurosciences, immunologie, microbiologie, pharmacologie, toxicologie, médecine clinique, santé publique.

${ }^{2}$ Mathématiques, physique, chimie, environnement, écologie, géosciences, ingénierie, sciences de l'agriculture, sciences de l'espace... 


\begin{tabular}{|c|c|c|c|c|c|}
\hline & \multirow[t]{2}{*}{ Publications } & \multirow[t]{2}{*}{ Citations } & \multirow[t]{2}{*}{ Citations/publications } & \multicolumn{2}{|c|}{ Top $1 \%$} \\
\hline & & & & Nombre & $\%$ \\
\hline \multicolumn{6}{|c|}{ Recherche médicale et biomédicale } \\
\hline États-Unis & 1546386 & 24649999 & 15,9 & 26803 & 1,7 \\
\hline Japon & 313325 & 3125478 & 9,8 & 1896 & 0,6 \\
\hline \multicolumn{6}{|l|}{ Europe des 15} \\
\hline 1. Grande-Bretagne & 329798 & 4480471 & 13,6 & 4392 & 1,3 \\
\hline 2. France & 215295 & 2588817 & 12,0 & 2060 & 1,0 \\
\hline 3. Allemagne & 297120 & 2270831 & 7,6 & 3006 & 1,0 \\
\hline 4. Italie & 152108 & 1691463 & 11,1 & 1529 & 1,0 \\
\hline 5. Pays-Bas & 107825 & 1506088 & 14,0 & 1381 & 1,3 \\
\hline 6. Suède & 87918 & 1186388 & 13,5 & 965 & 1,1 \\
\hline 7. Espagne & 86666 & 752657 & 8,7 & 573 & 0,7 \\
\hline 8. Belgique & 50508 & 662508 & 13,1 & 628 & 1,2 \\
\hline 9. Danemark & 41289 & 564568 & 13,7 & 505 & 1,2 \\
\hline 10. Finlande & 39654 & 539886 & 13,6 & 472 & 1,2 \\
\hline 11. Autriche & 37085 & 410437 & 11,1 & 363 & 1,0 \\
\hline 12. Irlande & 12872 & 131871 & 10,2 & 117 & 0,9 \\
\hline 13. Grèce & 17942 & 114430 & 6,4 & 95 & 0,5 \\
\hline 14. Portugal & 8176 & 69048 & 8,4 & 60 & 0,7 \\
\hline 15. Luxembourg & 526 & 4966 & 9,4 & 4 & 0,8 \\
\hline \multicolumn{6}{|c|}{ Recherche non biomédicale } \\
\hline États-Unis & 1253207 & 10718638 & 8,6 & 24461 & 2,0 \\
\hline Japon & 425883 & 2337807 & 5,5 & 3408 & 0,8 \\
\hline \multicolumn{6}{|l|}{ Europe des 15} \\
\hline 1. Allemagne & 382582 & 2703382 & 7,1 & 4994 & 1,3 \\
\hline 2. Grande-Bretagne & 289909 & 2121027 & 7,3 & 4460 & 1,5 \\
\hline 3. France & 286085 & 1922093 & 6,7 & 3117 & 1,1 \\
\hline 4. Italie & 171344 & 1070998 & 6,3 & 1702 & 1,0 \\
\hline 5. Pays-Bas & 94359 & 784972 & 8,3 & 1497 & 1,6 \\
\hline 6. Espagne & 132229 & 779966 & 5,9 & 964 & 0,7 \\
\hline 7. Suède & 70218 & 529303 & 7,5 & 961 & 1,4 \\
\hline 8. Belgique & 52673 & 342122 & 6,5 & 603 & 1,1 \\
\hline 9. Danemark & 38640 & 325450 & 8,4 & 661 & 1,7 \\
\hline 10. Finlande & 34452 & 213924 & 6,2 & 352 & 1,0 \\
\hline 11. Autriche & 34430 & 208598 & 6,1 & 420 & 1,2 \\
\hline 12. Grèce & 30400 & 133085 & 4,4 & 149 & 0,5 \\
\hline 13. Portugal & 20706 & 91347 & 4,4 & 126 & 0,6 \\
\hline 14. Irlande & 13395 & 71358 & 5,3 & 144 & 1,1 \\
\hline 15. Luxembourg & 28 & 75 & 2,7 & 0 & 0 \\
\hline
\end{tabular}

Tableau I. Production en recherche biomédicale et non biomédicale, de 1993 à 2003, des États-Unis, du Japon et des pays de l'Europe des 15. Les données présentées sont issues des bases de l'Institut for Scientific Information (Essential Science Indicators). Les chiffres sont des valeurs absolues excepté pour la dernière colonne qui donne le pourcentage de publications au Top $1 \%$. Chaque article compte pour chaque nation. Les articles au Top 1 \% (articles originaux et de synthèse) sont définis pour chaque année et chaque discipline (chaque article n'appartient qu'à une discipline). La recherche biologique et médicale comprend la biologie, la génétique, les neurosciences, l'immunologie, la microbiologie, la pharmacologie, la toxicologie, la médecine clinique et la santé publique ; la recherche non biomédicale comprend les mathématiques, la physique, la chimie, l'environnement, l'écologie, les géosciences, l'engénierie, les sciences de l'agriculture, les sciences de l'espace. Les publications comprennent les articles originaux, les articles de synthèse, les notes et les lettres. 


\section{Mesurer l'excellence}

Les publications scientifiques recouvrent deux réalités différentes, la nécessité de la communication vers les pairs et la nécessité du transfert de connaissance vers des utilisateurs qui ne font pas, stricto sensu, partie de la communauté scientifique. Les études qui se fondent uniquement sur le nombre total des publications - incluant celles publiées dans les revues nationales de faible notoriété à usage local ou didactique - ne s'appuient pas sur une mesure pertinente de la qualité scientifique. Cette dernière s'apprécie davantage par la capacité d'un pays de produire des travaux d'excellence dont l'impact permettra des avancées majeures.

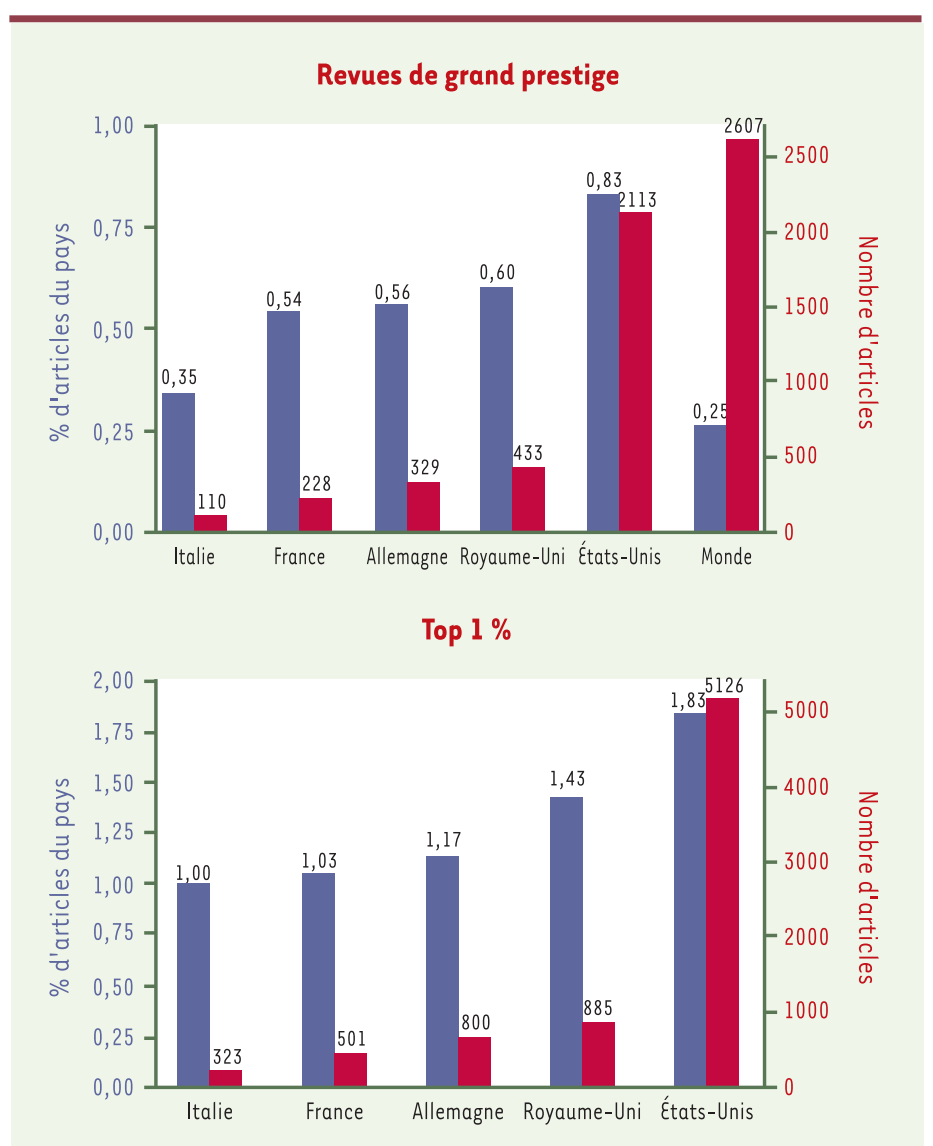

Figure 1. Production dans les revues de grand prestige du domaine biomédical (facteur d'impact supérieur à 20) et dans le corpus mondial d'excellence (Top $1 \%)$. Tous les calculs reposent sur les bases de données de l'Institute for Scientific Information (ISI) dans leur version Web, de 1999 à 2003 pour les revues de grand prestige et de 1993 à 2003 pour les revues Top $1 \%$. Les bases incluent le Science Citation Index, le Social Science Citation Index et Arts and Humanities Citation Index. Ces bases indexent les adresses complètes de tous les auteurs. Pour les données des journaux généralistes (New England Journal of Medicine, Science, Nature, Nature Genetics, Nature Immunology, Nature Cell Biology, Cell), seuls les articles sont comptabilisés (ni lettre, ni note), de même que pour les données totales correspondantes. Dans le cas des publications au Top $1 \%$, seuls les articles et les revues sont comptés (ni lettre, ni note).
Les articles les plus cités rapportent des découvertes exceptionnelles ou ayant un fort impact en terme de santé publique. À titre d'exemple, en médecine clinique, l'article le plus cité dans la période 1993-2003, est un essai thérapeutique, publié en 1995, sur la prévention des maladies cardiovasculaires. II a reçu 3109 citations $^{3}$ [3]. En biologie-biochimie, l'article le plus cité (excluant les descriptions de techniques) décrit la mise en évidence, en 1994, de la signalisation intracellulaire Jak-Stat par l'interféron. II a été cité 2346 fois $^{3}$ [4]. En sciences sociales-santé publique, l'article le plus cité (publié en 1994) a reçu 853 citations $^{3}$ [5]. Ces chiffres montrent la grande variabilité du nombre de citations en fonction des disciplines. Les articles extrêmement cités sont très rares, quelle que soit la discipline, et représentent moins de $0,01 \%$ du nombre total des articles publiés.

La mesure de l'excellence dans le monde scientifique ne fait pas l'unanimité [6-8]. Pour les uns, elle inclut le retour sur investissement, les licences et brevets, le nombre de molécules en développement, pour les autres, le nombre de prix Nobel, des médailles prestigieuses, le nombre d'articles dans les revues à très fort facteur d'impact ${ }^{4}$ (reflet de la sélectivité des éditeurs) et enfin le nombre d'articles au Top $1 \%{ }^{5}$ (reflet de la participation aux grandes découvertes). Néanmoins, les prix ou la valorisation sont toujours précédés d'une production de connaissance et les publications d'excellence en sont le témoin le plus précoce et le plus fidèle. Une étude récente montre que l'analyse des citations permet d'identifier la très grande majorité des travaux significatifs et que le nombre d'articles dont la reconnaissance par les pairs a été négligée dans un premier temps est extrêmement faible [9]. La production des articles les plus cités permet donc de suivre au plus près l'excellence d'un pays (ou d'un institut). Nous avons donc choisi d'analyser la place de la France en fonction de deux critères complémentaires : le nombre d'articles publiés dans les journaux de grande notoriété dont les articles sont majoritairement cités et le nombre d'articles au Top $1 \%$.

Les États-Unis publient $70 \%$ des articles ${ }^{6}$ des journaux de très fort facteur d'impact ${ }^{7}$ (supérieur à 20). Ils

\footnotetext{
${ }^{3}$ Citations en juin 2004

${ }^{4}$ Mesure du prestige du journal élaborée à partir du nombre moyen de citations de l'ensemble des articles sur une période de 2 ans.

${ }^{5}$ Pour chaque année et chaque discipline, les publications mondiales sont classées par nombre de citations reçues; ce classement permet ainsi d'identifier les articles les plus cités qui constituent le Top $1 \%$.

${ }^{6}$ Les articles écrits en collaboration sont comptés pour chaque pays.

${ }^{7}$ New England Journal of Medicine, Science, Nature, Nature Genetics, Nature Immunology, Nature Cell Biology, Cell (IF supérieur à 20) : 0,25\% des publications mon-
} 
occupent la première place suivis par le Royaume-Uni $(13,8 \%)$, l'Allemagne $(10,5 \%)$, la France $(7,3 \%)$ et I'Italie (3,5\%). Si on évalue la capacité d'un pays à publier dans ces revues dites de prestige (nombre d'articles publiés dans les revues à fort facteur d'impact/nombre total d'articles), la France se situe toujours à la $4^{\mathrm{e}}$ place mondiale $(0,54 \%)$, très près du score de l'Allemagne $(0,56 \%)$ et du RoyaumeUni $(0,60 \%)$. Le score des États-Unis est plus élevé (0,83\%).

Il est également possible d'identifier, grâce à la base internationale de données de $\left.\right|^{\prime} \mid S I^{8}$, les travaux les plus cités (Top $1 \%$ ) par nation, par institution, par chercheur et ceci dans chaque domaine ou discipline. Le corpus identifié ainsi par I'ISI contient, pour la période 1993-2003, 73280 articles toutes disciplines confondues. En nombre d'articles présents dans ce corpus, la France se situe à nouveau en $3^{\mathrm{e}}$ position dans l'Europe des 15 et à la $5^{\mathrm{e}}$ position mondiale. Les ÉtatsUnis ont $2 \%$ de leurs articles au Top $1 \%$, l'Angleterre 1,5\%, l'Allemagne 1,3\%, la France 1,1 \% et l'Italie 1\% (Figure 1).

\section{Analyse du corpus d'excellence biomédical de la France}

Pour la même période, 1993-2003, le corpus du Top 1 \% biomédical mondial rassemble 30700 publications. La sélection des articles ayant au moins une adresse française, permet d'identifier 1866 publications $(0,92 \%$ de l'ensemble des publications de la France et $6,07 \%$ du corpus). L'Allemagne en totalise 2604 $(0,96 \%$ de sa production ; 7,54 \% du corpus) et le Royaume-Uni 3895 (1,29\% de sa production et $11,27 \%$ du corpus) (Figure I).

Le corpus de publications associées à la France rassemble plus de 14000 auteurs, près de 4000 structures de recherche (adresses différentes). Il émane d'un millier de villes (françaises et étrangères) et de 70 pays. Les articles qu'il contient, très cités, ont été publiés pour $27 \%$, dans des revues de grand prestige ${ }^{7}$ et pour $73 \%$ dans les journaux de spécialités. La moyenne de citations est d'environ 200 par article. Vingt sont cités plus de 1000 fois et $65 \%$ plus de 100 fois.

Le nombre de publications associées à la France dans le corpus Top $1 \%$, entre 1993 et 2001 , s'accroît régulièrement. Cette évolution (70 \%) est presque exclusivement due aux publications de médecine clinique. L'augmentation de la totalité

${ }^{8}$ Institut for Scientific Information: base qui indexe plus de 8000 revues; entre 1993 et 2003, elle contient plus de 9 millions d'articles qui ont obtenu près de 53 millions de citations à la date de juin 2004 . des publications françaises dans la même période est de $40 \%$. Afin d'analyser plus finement les données françaises, d'identifier les disciplines majeures et les spécialités médicales les mieux représentées dans ce corpus d'excellence, un système d'indexation à trois niveaux: «grandes disciplines », «sous-disciplines » et «type de recherche », a été adopté. Chaque article n'a été assigné qu'à l'une des sept disciplines suivantes : biologie-biochimie, médecine clinique, génétique et biologie moléculaire, microbiologie (excluant les maladies infectieuses assignées à médecine clinique), neurosciences (incluant la psychiatrie, la psychologie, le comportement et la neurologie clinique) et santé publique. Bien que transversale, l'oncologie a été regroupée comme une spécialité unique de la médecine clinique.

Les articles de médecine clinique constituent le groupe le plus important du corpus (58\%). Ils ont été répartis en sous-disciplines. La plus représentée est l'oncologie (32\% des articles ), suivie des maladies infectieuses (17\%), de la cardiologie et des maladies cardiovasculaires (14\%), puis des maladies rares $(7 \%)$. Les autres sous-disciplines représentent moins de $5 \%$. Pour ce qui concerne les types de recherche, $45 \%$ des articles traitent des

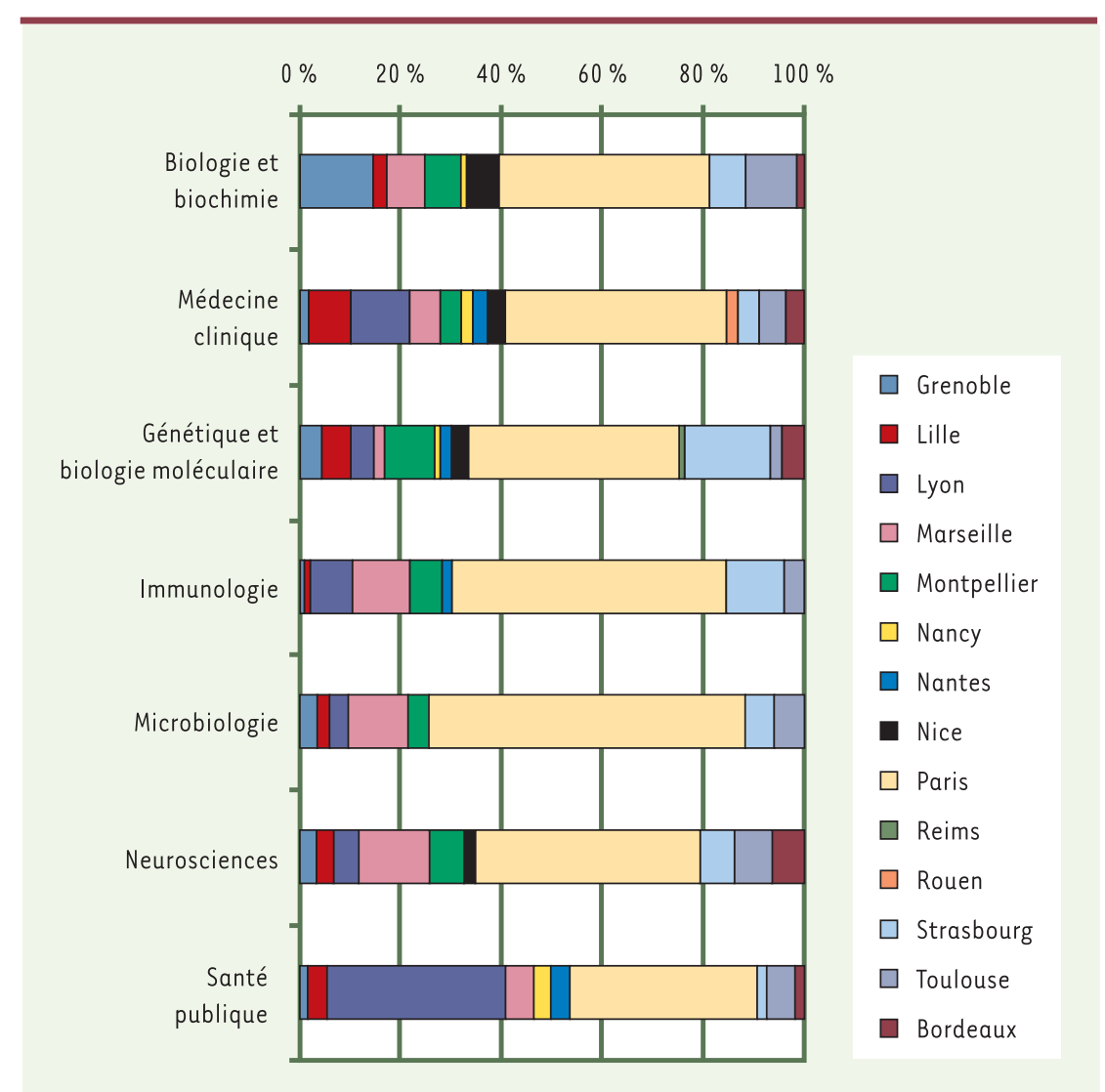

Figure 2. Participation des sites de recherche des grandes villes françaises aux sept domaines de la recherche biomédicale. 
recherches fondamentales, $21 \%$ des essais cliniques ou investigations cliniques, $13 \%$ d'études épidémiologiques et moins de $5 \%$ des techniques. Treize pour cent sont des revues de synthèse.

\section{Répartition régionale}

Selon les données de l'Observatoire des Sciences et de la technologie [1] l'île-de-France est associée, en 2000 , à $40 \%$ des publications biomédicales françaises. Nos données indiquent qu'elle totalise plus de $50 \%$ des publications au Top $1 \%$ dans le même domaine. Paris (banlieues proches comprises) est majoritairement associée aux publications du Top $1 \%$ (42\% des articles). La deuxième position revient à Lyon (10\%), suivi de Marseille (7\%), Lille (6\%), Strasbourg (5,5\%) et Toulouse (5\%). Pour les 15 villes analysées, le domaine le plus représenté est la médecine clinique. Le deuxième domaine varie selon les villes, génétique à Strasbourg, santé publique à Lyon, biologie-biochimie à Grenoble, neurosciences à Paris,

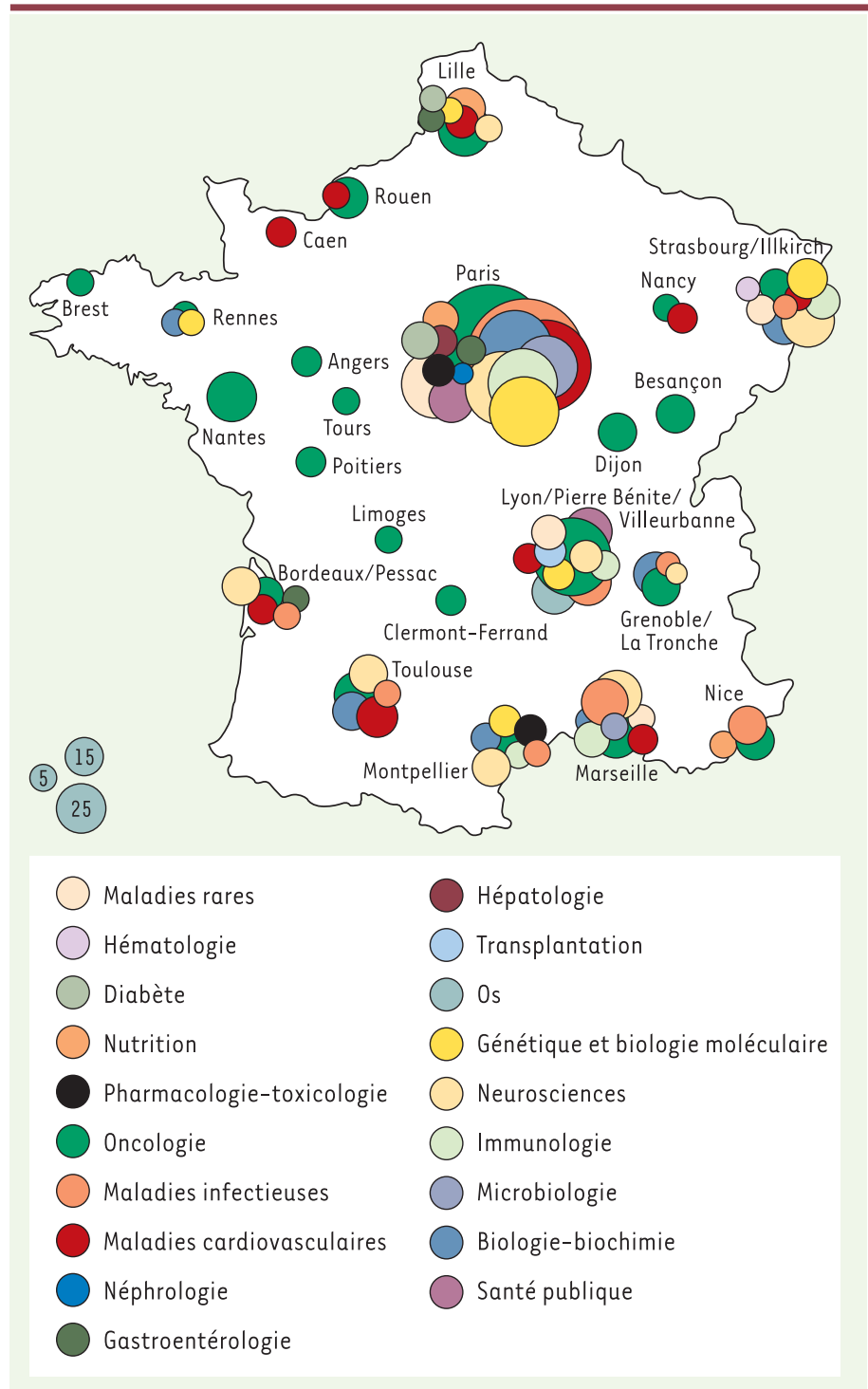

Figure 3. Sites de France qui totalisent au moins cinq articles au Top $1 \%$ pour une discipline ou sous-discipline, tous organismes de recherche confondus.
Bordeaux, Montpellier et Marseille (Figure 2). L'analyse plus détaillée du domaine de la médecine clinique indique que dans toutes les villes, sauf Nancy, I'oncologie arrive en tête. Puis, selon les villes, maladies infectieuses ou cardiologie et maladies vasculaires sont en deuxième position. Lille est un site d'excellence pour les recherches en nutrition et sur le diabète ; Paris pour les maladies rares ${ }^{9}$, Marseille et Nice pour les maladies infectieuses (Figure 3).

\section{Collaborations internationales et nationales}

Le degré de collaboration le plus élevé s'établit avec les États-Unis qui cosignent $36 \%$ des articles du corpus Top $1 \%$ de la France, puis avec le Royaume-Uni (21\%), l'Allemagne ( $16 \%$ ) et l'Italie (12\%). Il varie en fonction du type d'article : dans le cas des recherches fondamentales, les investigations cliniques et les essais cliniques de phases I et II, une grande part des travaux d'excellence sont issus d'équipes uniquement françaises (environ $50 \%$ ). En revanche, dans le cas des essais cliniques de phases III et IV, les équipes françaises sont insérées dans les réseaux internationaux d'excellence ( $80 \%$ des articles sont issus de collaborations).

Le site Paris est plus associé à des villes étrangères qu'à des villes nationales avec un axe fort Paris-Londres (10\% des articles associés à Paris) suivi de Lyon, New York, Boston, Bruxelles, avant les autres villes françaises. Le site Lyon est associé à Paris puis à Londres et à Bethesda. Les autres grandes villes françaises copublient prioritairement avec Paris, puis avec d'autres sites régionaux. Les axes majeurs s'établissent entre Lyon et Marseille ou Toulouse, entre Strasbourg, Lyon et Marseille, entre Bordeaux, Lyon et Marseille donnant après Paris, un rôle clé à Lyon.

\section{L'université et l'hôpital}

La recherche biomédicale associe les acteurs du monde universitaire et hospitalier. Les centres hospitaliers et l'université vivent de longue date en symbiose et ils sont des acteurs majeurs dans le développement de la recherche clinique.

En région parisienne ${ }^{10}$, le site de la Pitié-Salpêtrière (104 publications au Top $1 \%$ - plus de 10 par an) est reconnu pour ses articles sur les maladies infectieuses, les neurosciences, les maladies rares, les maladies cardiovasculaires et l'oncologie. Le site Necker-Enfants Malades (93 publications) s'illustre par ses travaux sur

\footnotetext{
${ }^{9}$ Indexées selon la classification Orphanet-maladies rares, génétiques ou avec une susceptibilité génétique.

${ }^{10}$ Sites identifiés par au moins trois spécialités ayant au moins cinq articles dans le
} Top $1 \%$. 
les maladies rares, l'immunologie, les maladies infectieuses, l'oncologie et la néphrologie. Le site Saint-Louis (69 publications) a pour spécialités médicales majeures l'oncologie, la transplantation et le diabète. Le site de Cochin-Port Royal (57 publications) figure dans le Top $1 \%$ pour ses articles sur les maladies infectieuses, les soins intensifs et les maladies osseuses (Figure 4).

$\varepsilon$ n province, les sites des centres hospitalo-universitaires (CHRU) s'identifient majoritairement par deux spécialités médicales: l'oncologie et les maladies cardiovasculaires. Outre ces spécialités, le CHRU de Lyon (78 publications au Top $1 \%$ ) présente des travaux remarquables sur les maladies osseuses, I'hépatite infectieuse et l'immunologie. Le CHRU de Lille (54 publications) est reconnu pour ses travaux en nutrition, celui de Toulouse ( 45 publications au total) pour ses études en neurosciences, celui de Bordeaux (37 publications) pour ses études en gastro-entérologie (Figure 4).

\section{Les organismes de recherche}

Les organismes de recherche français très fortement impliqués dans la recherche biomédicale, l'Inserm, le CNRS (sciences du vivant) et l'Institut Pasteur, sont signataires de plus de la moitié des articles du Top $1 \%$. L'Inserm (593 publications) est en $1^{\text {re }}$ position pour la recherche en médecine clinique, en neurosciences et en immunologie, le CNRS (520 publications) est en $1^{\text {re }}$ position en biologie-biochimie et génétique-biologie moléculaire. L'Institut Pasteur (198 publications) est premier en microbiologie et maladies infectieuses. Les spécialités médicales phares de l'Inserm sont l'oncologie, les maladies infectieuses (toutes maladies confondues), les maladies rares et les maladies cardiovasculaires. Celles du CNRS sont l'oncologie, les maladies infectieuses et la pharmacologie-toxicologie. Environ $30 \%$ des publications au Top $1 \%$ du CNRS et de l'Inserm sont cosignées par les deux organismes. L'Institut Pasteur cosigne $40 \%$ de ses articles au Top $1 \%$ avec le CNRS et $35 \%$ avec I'Inserm.

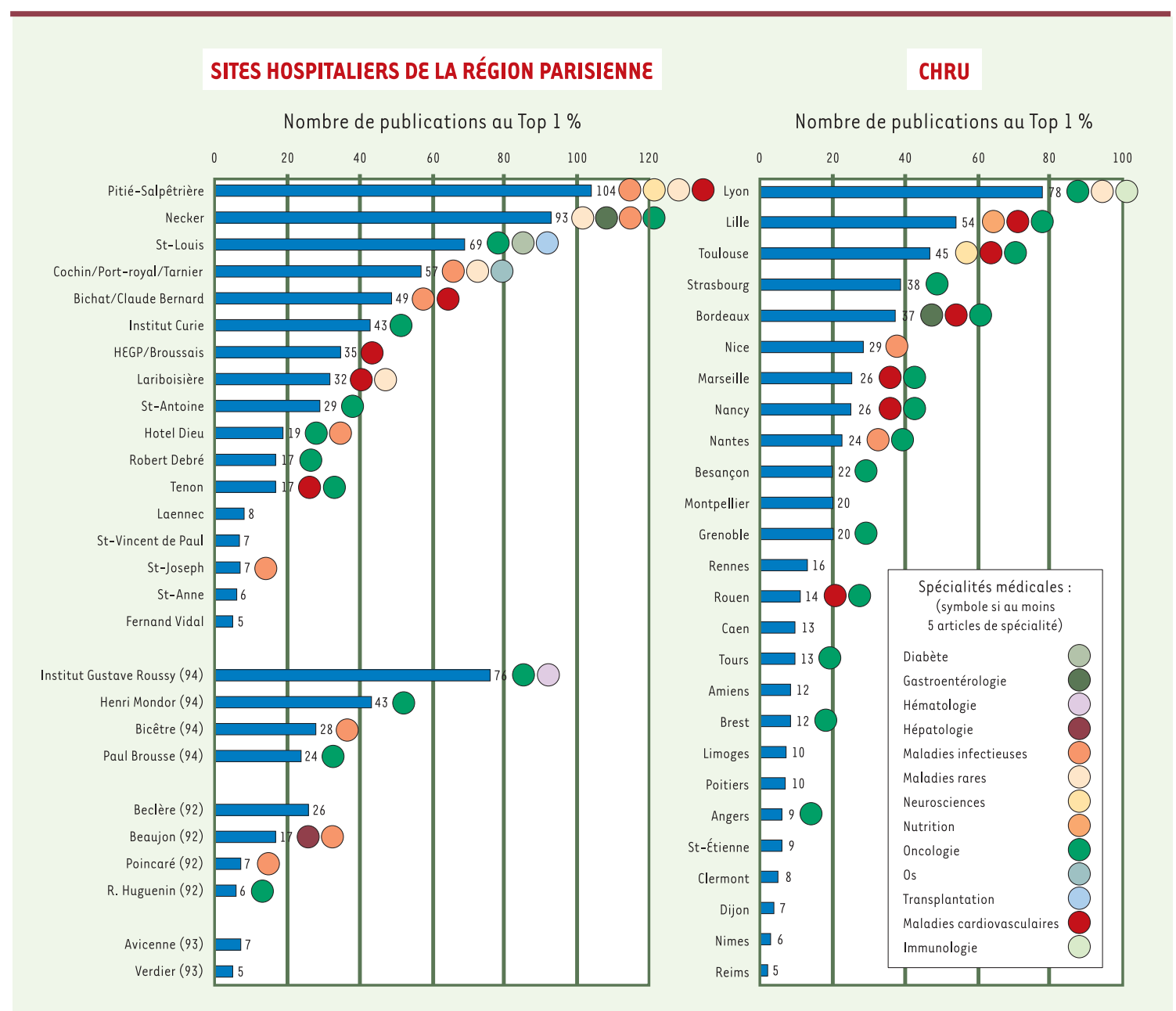

Figure 4. Sites hospitaliers de la région parisienne et les autres centres hospitalo-universitaires régionaux (CHRU) qui totalisent au moins 5 publications au Top $1 \%$. 


\section{Conclusions}

La communication scientifique est un phénomène socioculturel complexe, influencée par une multitude de facteurs d'ordre économique, sociétal, psychologique... La production des publications scientifiques est la «vitrine » la plus visible de ce processus et fait l'objet de nombreuses études bibliométriques. Les données bibliographiques y compris les citations deviennent facilement disponibles aujourd'hui grâce aux bases de données sur Internet. La facilité apparente de leur maniement peut conduire à utiliser des critères réducteurs ne mesurant qu'un paramètre pour en tirer des conclusions globales. Les experts en bibliométrie s'entendent sur la nécessité d'utilisation d'indicateurs multiples comme seul moyen d'approcher la réalité de façon adéquate [10]. Quels que soient les indicateurs utilisés, la France se situe parmi les grandes nations scientifiques. Elle est présente dans les travaux d'excellence et ses chercheurs contribuent aux grandes avancées de la science. Au cours de ces dix dernières années, un grand nombre de ses travaux a été publié dans les meilleurs journaux scientifiques et les découvertes ont été relayées par la communauté scientifique mondiale comme en attestent les citations reçues. Sa participation au corpus des articles d'excellence du Top $1 \%$ et sa capacité de publier dans les journaux de plus haut niveau augmente régulièrement avec actuellement plus de 200 articles par an dans les revues prestigieuses et plus de 500 par an au Top $1 \%$ dont près de 200 dans le domaine biomédical. $\diamond$

\section{SUMMARY}

Zoom on the excellence of the biomedical research in France Among the key benchmarks in assessing research excellence is the production and recognition of scientific discoveries for innovation. Despite the growing use of bibliometric indicators for policymaking purposes, there is still no consensus concerning the appropriate measures of research excellence. In this study, we examine the performance of France in biomedical sciences using several ISI-based indicators. We focus on the results provided by these two selective indicators: the absolute numbers and proportion of papers published in the very high-impact journals (above 20) and in the $1 \%$ of the most highly cited papers. Furthermore, we present the detailed analysis of the Top $1 \%$ French biomedical articles. On this basis we identify the crucial fields, the most active centres per speciality and the networks and the degree of international collaboration resulting from different types of research. These results provide an objective demonstration that the French biomedical research meets with high international standards and contributes to the world core research. $\diamond$

\section{RÉFÉRENCES}

1. Observatoire des sciences et des techniques. Science et technologie, indicateurs. Paris: Economica, $2000: 512 \mathrm{p}$.

2. Centre d'études de la science et de la technologie. Scientometrics scoreboard 2003. http://www.cest.ch/Publikationen/2003/Diverse-Doks/web-scoreboard-fr12-03.pdf

3. Shepherd J, Cobbe SM, Ford I, et al. Prevention of coronary heart-disease with pravastatin in men with hypercholesterolemia. N Engl J Med 1995 ; 333 : 1301-7.

4. Darnell JE, Kerr IM, Stark GR. Jak-Stat pathways and transcriptional activation in response to Ifns and other extracellular signaling proteins. Science $1994 ; 264$ : 1415-21.

5. McHorney CA, Ware JE, Lu JFR, et al. The mos 36-item short-form health survey $(\mathrm{Sf}-36)$. 3. Tests of data quality, scaling assumptions, and reliability across diverse patient groups. Med Care $1994 ; 32: 40-66$.

6. Les États-Unis loin devant l'Europe dans le domaine des sciences du vivant. Le Figaro 3 juin 2004.

7. Shanghai Jiao Tong University. Academic ranking of world universities, 2003. http://ed.sjtu.edu.cn/ranking.htm

8. Blackman S. The right research mix. Scientist $2004 ; 18: 17-21$. http://www.thescientist.com/yr2004/mar/feature_040301.html

9. Glanzel W, Schlemmer B, Thijs B. Better late than never? On the chance to become highly cited only beyond the standard bibliometric time horizon. Scientometrics $2003 ; 58: 571-86$.

10. Van Leeuwen TN, Visser MS, Moed HF, et al. Holy grail of science policy: exploring and combining bibliometric tools in search of scientific excellence. Scientometrics $2003 ; 57: 257-80$.
TIRÉs À PART

N. Haeffner-Cavaillon 